\title{
KMD clustering: Robust generic clustering of biological data
}

\author{
Aviv Zelig ${ }^{1,2}$ and Noam Kaplan², \\ 1 - Data Science \& Engineering Program, Faculty of Industrial Engineering \& Management, \\ Technion - Israel Institute of Technology \\ 2 - Department of Physiology, Biophysics \& Systems Biology, Rappaport Faculty of Medicine, \\ Technion - Israel Institute of Technology \\ * - corresponding author
}

Corresponding author email: noam.kaplan@technion.ac.il 


\section{Abstract}

The challenges of clustering noisy high-dimensional biological data have spawned advanced clustering algorithms that are tailored for specific subtypes of biological datatypes. However, the performance of such methods varies greatly between datasets, they require post hoc tuning of cryptic hyperparameters, and they are often not transferable to other types of data. Here we present a novel generic clustering approach called k minimal distances (KMD) clustering, based on a simple generalization of single and average linkage hierarchical clustering. We show how a generalized silhouette-like function is predictive of clustering accuracy and exploit this property to eliminate the main hyperparameter $\mathrm{k}$. We evaluated KMD clustering on standard simulated datasets, simulated datasets with high noise added, mass cytometry datasets and scRNA-seq datasets. When compared to standard generic and state-of-the-art specialized algorithms, KMD clustering's performance was consistently better or comparable to that of the best algorithm on each of the tested datasets. 


\section{Introduction}

Clustering is a ubiquitous set of machine learning techniques that are widely used in data analysis to computationally group sets of objects based on some measure of pairwise distance or similarity. The application of clustering to complex biological datasets is widespread ${ }^{1-6}$ with some notable recent examples including the clustering of single cell RNA sequencing ${ }^{7}$ (scRNA-seq) and mass cytometry data ${ }^{8}$ with the aim of detecting cell subpopulations. In biological applications, underperformance of standard generic clustering algorithms on noisy high-dimensional data has led to the development of clustering algorithms that specialize in specific subtypes of biological data. While these algorithms outperform generic clustering algorithms on these datasets, they are often not transferrable to other types of data and their performance can vary significantly even on data of the same type ${ }^{9,10}$. Several examples of such specialized clustering methods exist, based on a variety of approaches. In SCRNA-seq clustering of cells, SCAAF ${ }^{11}$ uses a self-projection machine learning approach on a pre-clustered dataset to simultaneously identify distinct cell groups and a weighted list of feature genes for each group. In mass cytometry, FlowSOM ${ }^{12}$ clusters the nodes of a constructed self organizing map (SOM) connected by a minimal spanning tree using consensus hierarchical clustering. An exception to these specialized methods is Phenograph ${ }^{13}$ (other implementations include Scanpy ${ }^{14}$ Louvain and Seurat ${ }^{15}$ Louvain), which uses a shared neighbor graph to perform Louvain community detection and can be used on different types of biological datasets.

An important issue with many modern clustering methods, including the aforementioned specialized clustering algorithms, is the requirement of user-specified numerical hyperparameters. Although the values of such hyperparameters can change the clustering results dramatically ${ }^{1}$, they are usually cryptic, in the sense that they do not have a sufficiently clear interpretation such that a user would be able to set them a priori. Ultimately this leaves users to either use inadequate default hyperparameter settings or to adjust the hyperparameter values until they are happy with the outcome, which may lead to undetectably biased results and overfitting. For example, SCAAF is dependent on the self-projection machine learning parameters, clustering parameters and minimum self-projection accuracy parameter ${ }^{11}$; FlowSOM has several parameters regarding starting population, clustering channels and SOM settings ${ }^{12}$; Phenograph parameters include a dimension parameter for dimensionality reduction and the number of nearest neighbors when constructing the cell contact graph ${ }^{13}$. Ideally, one would like a clustering algorithm to either not have such hyperparameters or have hyperparameters that are easy to set (e.g. interpretable and do not significantly affect the results). 
Here we present a new generic clustering method which we call k-minimal distances (KMD) clustering. Our method is based on a natural generalization of average and single linkage, within the framework of hierarchical clustering. Building on this generalized linkage, we demonstrate its efficient computation and introduce a simple outlier-aware partitioning scheme in order to improve performance in noisy scenarios. Next, we propose a generalized silhouette-like function which corresponds to our generalized linkage, and show that it is predictive of clustering performance across different values of the hyperparameter k. Using this, we are able to eliminate the need to choose a value for the cryptic hyperparameter k. Finally, we apply our method to a range of simulated and biological datasets in which ground truth is known and find that our method compares favorably to both standard generic algorithms and domain-specific state-of-the-art algorithms.

\section{Results}

\section{KMD linkage}

We sought to generalize the notion of single and average linkage in order to capture the best aspects of both within the framework of hierarchical clustering (Figure 1a). Hierarchical clustering starts with $n$ clusters of size one and then iteratively merges the two nearest (most similar) clusters. However, since only distances between pairs of objects are given, one must define how to calculate the distances between any two clusters, and this is known as linkage. In single linkage, the distance between two clusters is defined as the minimal pairwise inter-cluster distance. Single linkage can detect both globular and complex non-globular cluster shapes, but is highly prone to noise due to its reliance on a single pairwise distance ${ }^{16}$. In average linkage, the distance between two clusters is defined as the average of all pairwise inter-cluster distances ${ }^{1718}$. Average linkage clustering is more robust to noise than single linkage ${ }^{16}$, but is biased towards detecting globular clusters ${ }^{18}$. We propose a generalized form of linkage which we refer to as $k$ minimal distances linkage (KMD linkage), in which we define the distance between two clusters as the average of the $k$ minimal pairwise inter-cluster distances, where $k$ is an integer (Figure 1a). Note that $k=1$ gives single linkage and $k>>n$ gives average linkage. Thus, an intermediate value of $k$ might potentially be more robust to noise than single linkage, yet less biased towards globular clusters than average linkage. As we show later, prior knowledge of the value of $k$ is not needed since $k$ can be estimated computationally. While the implementation of a hierarchical clustering algorithm with an arbitrary linkage function can be computationally impractical, efficient algorithms for single and average linkage clustering are known. Similarly, for KMD linkage we show a method for efficient linkage update in linear time, as well as a quadratic memory 
implementation which is importantly independent of $k$, making computations with large $k$ feasible (see Methods).

\section{Fig.1}
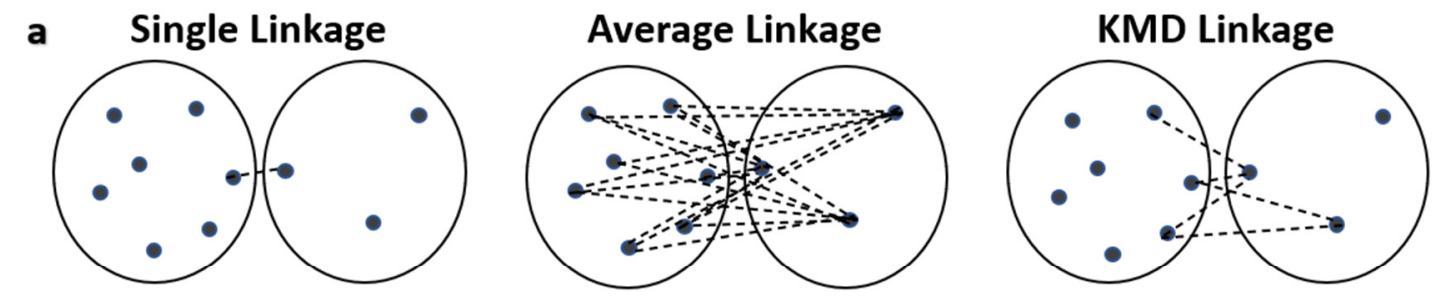

$\min \{d(a, b): a \in A, b \in B\}$

$$
\frac{1}{|A| *|B|} \sum_{a \in A} \sum_{b \in B} d(a, b) \quad \frac{1}{k} \sum \min _{k}\{d(a, b): a \in A, b \in B\}
$$

b
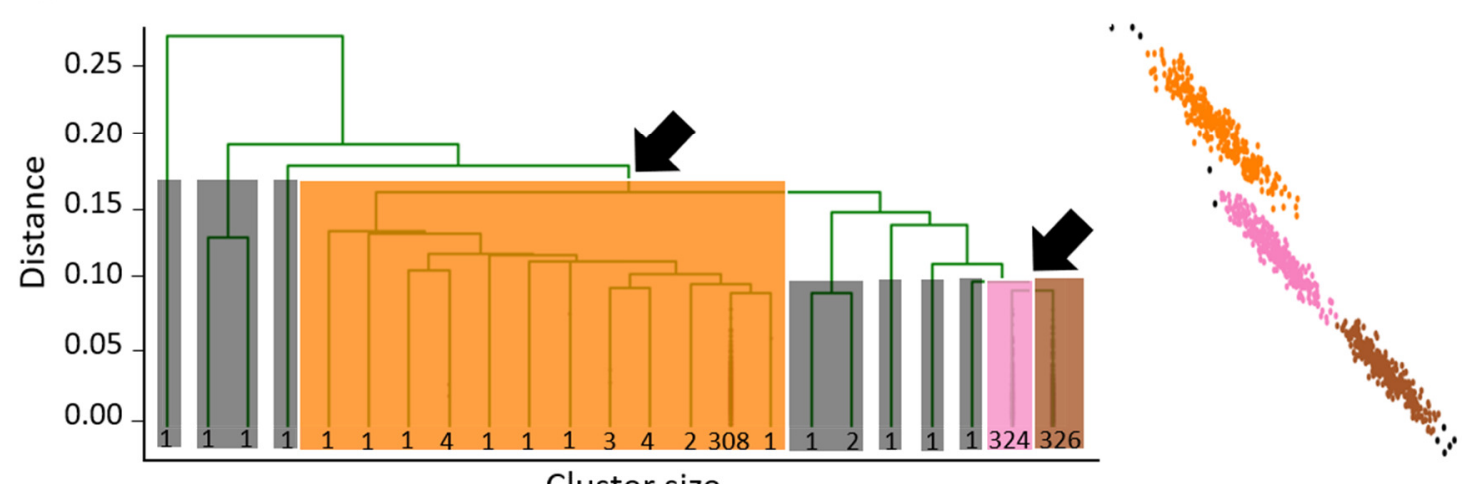

Cluster size

Figure 1. KMD clustering. (a) Illustration of single, average and KMD linkage methods. (b) Outlier-aware partitioning schematic example (Left: dendrogram; Right: data points). Orange, pink and brown indicate core clusters; grey rectangles/points represent outliers; black arrows indicate merges between core clusters.

\section{Outlier-aware partitioning}

In order to partition the data into $c$ clusters in standard single and average linkage clustering, the clustering (merging) process is stopped when $c$ clusters are left ${ }^{19}$. However, in noisy datasets where an unknown amount of outlier objects may exist in addition to the $c$ clusters, outliers could be merged towards the end of the clustering process, leading to incorrectly merged clusters under the standard partitioning approach.

To address this challenge, we implemented a simple outlier-aware partitioning scheme (Figure 1b). We assumed that in the final stages of the partitioning, merges of tiny clusters are indicative 
of outlier clusters. Thus, our scheme ignores such merges in the final clustering steps and maintains only the last $c$-1 merges between non-tiny clusters. Tiny clusters (smaller than a given size) are then considered to be outliers, and the remaining $c$ clusters are considered as core clusters. Thus, the outlier cluster size threshold would be set by the user to be smaller than the minimal expected cluster size. Additionally, we find that clustering performance is typically robust across a large range of threshold values (e.g. high-noise half moons dataset, see "Evaluation on simulated datasets" for details, Figure S1).

As it still may be useful to associate the outliers to one of the core clusters, for example when comparing predictive performance, we calculate the KMD linkage of an outlier to each of the core clusters and assign it to the core cluster with the minimal distance. In the interest of fairness, all comparisons of clustering performance in the paper were done using these outlier assignments, such that no objects were left out unless explicitly stated otherwise. Finally, we assign each outlier classification a confidence score such that 0.5 is the lowest confidence assignment and 1 is the highest confidence assignment (Figure S2).

\section{Estimation of hyperparameter $\mathrm{k}$ by KMD silhouette}

Due to the drawbacks of cryptic hyperparameters, we asked whether it is possible to eliminate the main hyperparameter $k$. We first examined the effect of the hyperparameter $k$ on our clustering method. The hyperparameter $k$ plays a vital role in the method, as small $k$ values increase sensitivity to noise and large $k$ values favor globular clusters. While testing the clustering accuracy for a given dataset across different values of $k$, we found that often neither $k=1$ (single linkage) nor $k>>n$ (average linkage) give the best solution, and that clustering performance can vary dramatically for different values of $k$ (high-noise half moons dataset, see "Evaluation on simulated datasets" for details, Figure 2).

In order to eliminate the hyperparameter $k$, we asked whether there exists some intrinsic property which may indicate the quality of a clustering solution, thus allowing hyperparameter $k$ to be chosen automatically. A common method for measuring the quality of a clustering solution is the silhouette score, which is based on comparing each object's intercluster vs intracluster distances ${ }^{20}$. However, due to its similarity to the average linkage calculation, the silhouette score tends to favor globular clusters and thus will generally not be indicative of clustering performance when clusters are non-globular. To overcome this limitation, we propose a generalized modified form of the silhouette score which matches the KMD linkage. This new generalized function, which we refer to as KMD silhouette, calculates the difference between an object's intercluster and 
intracluster distances by only using the $k$ minimal distances, where $\mathrm{k}$ is selected to match the $k$ value used for clustering. In addition, we add a factor that penalizes large values of $k$. Thus, the calculation of the KMD silhouette changes for different values of $k$. Next, we tested whether the intrinsic KMD silhouette measure that we proposed is predictive of the actual clustering performance when compared to ground truth across different values of $k$ (high-noise half moons,

Figure 2). Remarkably, we find that KMD silhouette is highly predictive of the clustering accuracy (Pearson correlation $=0.987, \mathrm{n}=100$ ), in spite of it being calculated differently for different values of $k$. Thus, in KMD clustering, the hyperparameter $k$ is practically eliminated by running the clustering in parallel over several values of $k$ and picking the $k$ value that has the highest KMD silhouette score.

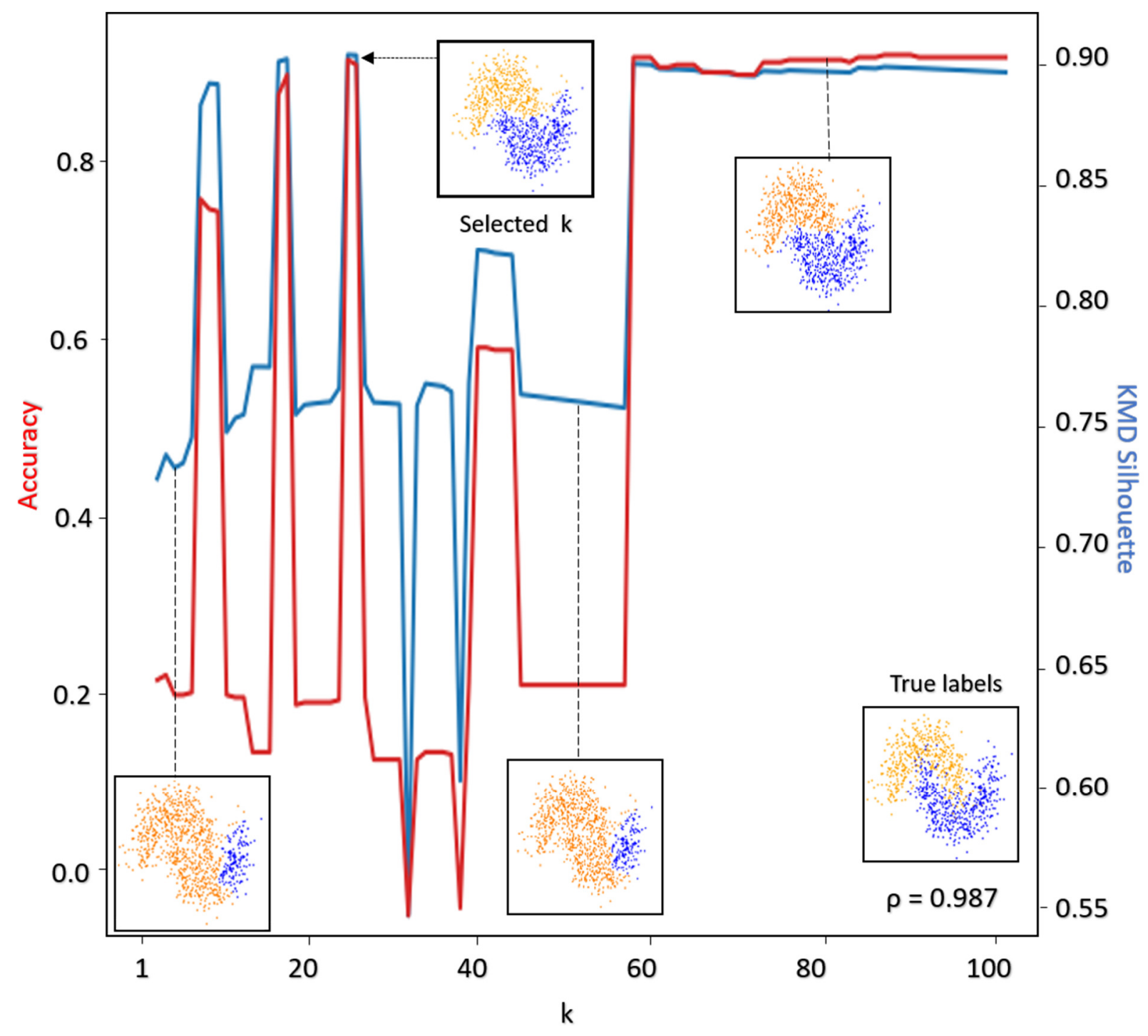


Figure 2. Selection of hyperparameter $\boldsymbol{k}$. KMD clustering accuracy (red line) and KMD silhouette score (blue line) on the high-noise half moons dataset across a range of $k$ values (1-100). Insets show true labels as well as cluster assignments at $k$ values of 1, 40,50 and 80. Pearson correlation between the accuracy and KMD silhouette score is shown in the bottom right corner.

\section{Evaluation on simulated datasets}

We first sought to evaluate and characterize our method's performance on a standard set of simulated datasets, provided by the python package scikit-learn ${ }^{21}$ to demonstrate the strengths and weaknesses of standard generic clustering algorithms. The scikit-learn datasets consist of five two-dimensional clustering problems (nested circles, half moons, globular clusters, and anisotropic clusters; each containing 1000 datapoints), which are generated from a mathematical function with a parameter controlling the amount of variance/noise. Each clustering problem is given the true cluster labels, allowing quantitative evaluation of clustering performance. For each clustering problem, we ran six different standard clustering algorithms, as well as KMD clustering. We quantified performance using three standard performance metrics: accuracy, Normalized Mutual Information (NMI) and Adjusted Rand Index (ARI), in which the highest achievable score is 1 (see Methods for details). We find that in each of the clustering problems, KMD clustering is either highly competitive or outperforms the other approaches (Figure 3, Table S1). Comparing performance across all datasets, KMD clustering achieves high performance scores on all datasets (nested circles: Accuracy=1/NMI=1/ARI=1, half moons: $1 / 1 / 1$, globular clusters: $0.961 / 0.847 / 0.888$, anisotropic clusters: 0.995/0.974/0.985), while spectral clustering which is the second best achieves comparable scores on three of the datasets but mislabels a patch at the edge of one of the anisotropic clusters (0.949/0.838/0.853). In addition, we asked whether the core clusters are identified with higher accuracy than the detected outliers, and indeed we observed that in all cases the performance metrics are higher when ignoring detected outliers, validating our outlier detection method, that correctly excludes irregular data objects. Taken together, we find that our method is the only method amongst those tested, which performs well on all tested clustering problems. 


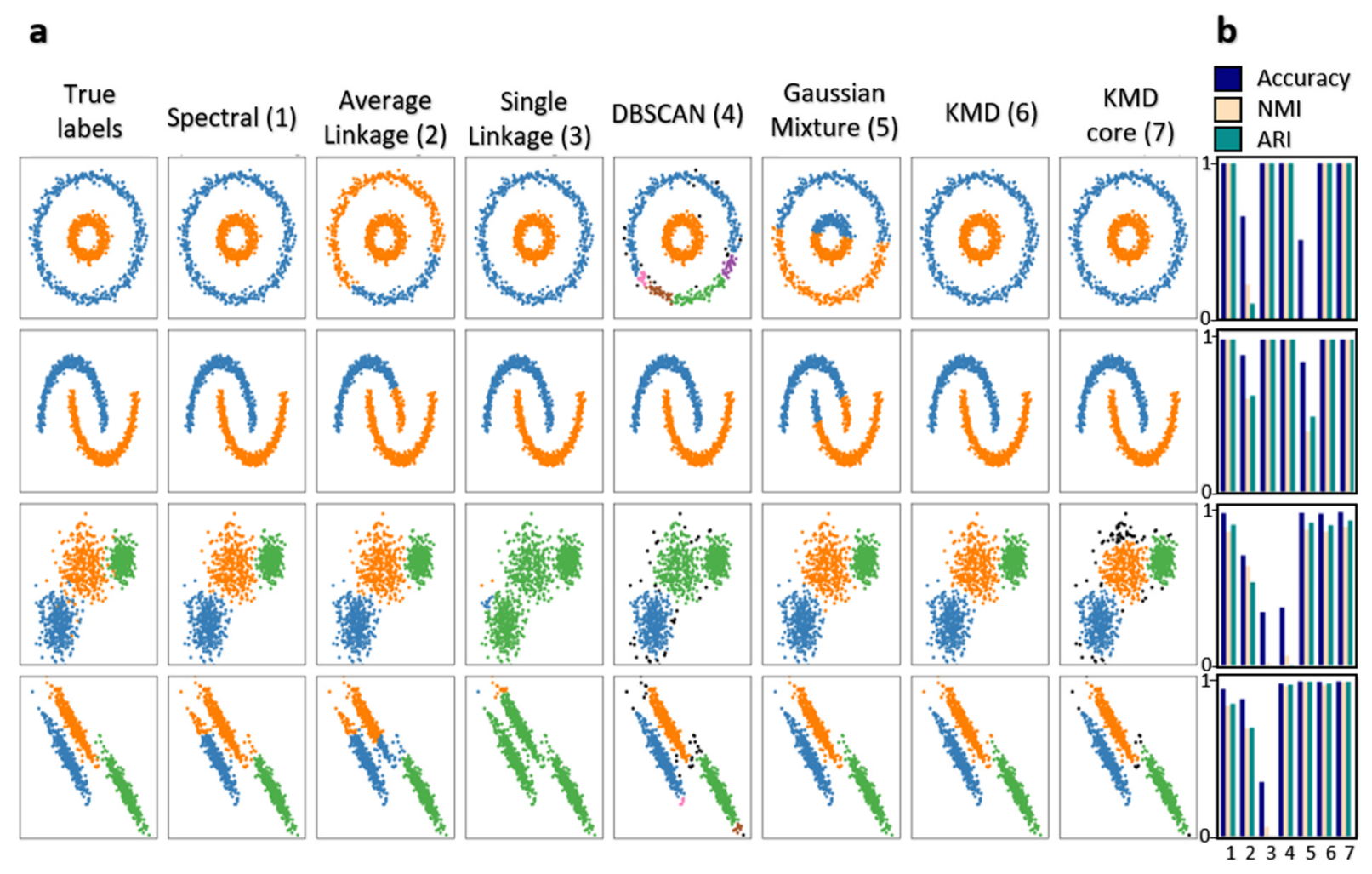

Figure 3. Evaluation on simulated datasets. (a) Comparison of clustering algorithm performance on standard scikit-learn simulated datasets (top to bottom: nested circles, half moons, globular clusters, anisotropic clusters). Algorithms (left to right): Spectral clustering (1), hierarchical average linkage (2), single linkage (3), DBSCAN (4), gaussian mixture (5), KMD clustering (6) and KMD clustering core clusters (outliers shown in black) (7). Datasets (top-down): nested circles, half moons, globular clusters and anisotropic clusters. (b) Evaluation of algorithms by accuracy (blue), Normalized Mutual Information (light pink), Adjusted Rand Index (green).

As one of our goals was to develop a method that performs robustly in noisy clustering problems, we tested our algorithm on the scikit-learn datasets with extreme noise added, such that in some cases the clusters were even difficult to resolve visually (Figure 4). We then reevaluated each of the clustering algorithms on these noisy datasets (Table S2). Remarkably, we find that KMD clustering identified correctly both irregular and gaussian cluster shapes (accuracy - nested circles: 0.989; half moons: 0.933; globular clusters: 0.909; anisotropic clusters: 0.992). The second best algorithm was gaussian mixture, which identified gaussian cluster shapes but not irregular cluster shapes (accuracy - globular clusters: 0.923, anisotropic clusters: 0.996; nested circles: 0.555 , half moons: 0.834). Taken together, we find that our method is the only method amongst those tested, which performed well on all four noisy clustering problems. We conclude 
bioRxiv preprint doi: https://doi.org/10.1101/2020.10.04.325233; this version posted October 4, 2020. The copyright holder for this preprint (which was not certified by peer review) is the author/funder, who has granted bioRxiv a license to display the preprint in perpetuity. It is made available under aCC-BY-NC-ND 4.0 International license.

that the KMD algorithm consistently outperforms standard general clustering algorithms across different simulated low-dimensional datasets, including extreme noise scenarios.

a

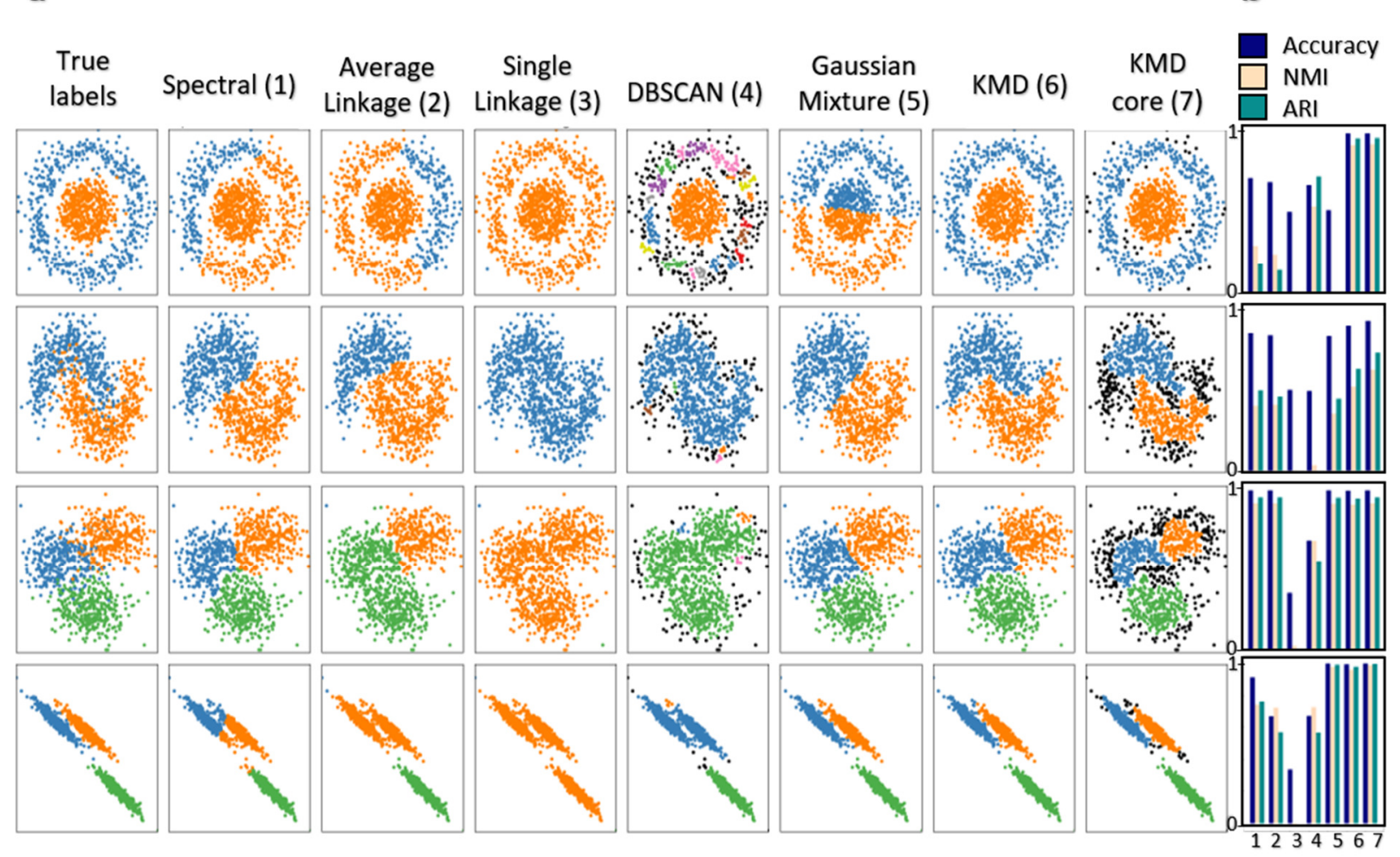

Figure 4. Evaluation on simulated high noise datasets. (a) Comparison of clustering algorithm performance on standard scikit-learn simulated datasets with added high noise (see Methods for details). Algorithms (left to right): Spectral clustering (1), average linkage (2), single linkage (3), DBSCAN (4), gaussian mixture (5), KMD clustering (6) and KMD clustering core clusters (outliers shown in black) (7). Datasets (top-down): nested circles, half moons, globular clusters and anisotropic clusters. (b) Evaluation of algorithms by accuracy (blue), Normalized Mutual Information (light pink), Adjusted Rand Index (green).

\section{Evaluation on mass cytometry data}

Next, we asked how KMD clustering will perform on a complex high-dimensional biological datatype. To this end, we considered the problem of clustering mass cytometry data. In mass cytometry, dozens of molecular markers are measured for each cell within a sample, and we seek to identify clusters representing cell populations. To assess the performance of KMD clustering in an unbiased manner, we followed the work of Liu et al ${ }^{10}$, which benchmarked several state-ofthe-art clustering algorithms. Liu et al ${ }^{10}$ use three bone marrow mass cytometry datasets for which 
the true labels are known, and repeatedly sample 20,000 cells from each of these datasets (Levine15_13: 20,000 cells out of 167,044, 13 markers, 14 clusters; Levine15_32: 20,000 cells out of 265,627, 32 markers, 24 clusters; Samusik16: 20,000 cells out of 86,864, 44 markers,24 clusters). We then compared the performance of KMD clustering to leading algorithms that were specifically designed for clustering mass cytometry data Xshift, DEPECHE, Accense, FlowSOM as well as the general clustering algorithms kmeans and Phenograph (Figure 5, Table S3). In order to ensure consistency and correct usage of the algorithms, we did not rerun the algorithms but took the performance results directly from Liu et $\mathrm{al}^{10}$. On the Levine15_32 dataset, we find that KMD clustering outperforms all other tested clustering methods (KMD: 0.945 accuracy, 0.935 NMI, $0.966 \mathrm{ARI}$; DEPECHE (second best): 0.892 accuracy, $0.842 \mathrm{NMI}, 0.927 \mathrm{ARI}$ ). On the Levine15_13 dataset, we also find that KMD clustering outperforms all other tested clustering methods (KMD: 0.883 accuracy, $0.875 \mathrm{NMI}, 0.865 \mathrm{ARI}$; FlowSOM (second best): 0.843 accuracy, 0.844 NMI, $0.858 \mathrm{ARI}$; PhenoGraph achieves 0.918 accuracy, but $0.139 \mathrm{NMI}$ ). On the Samusik16 dataset, KMD clustering performance was approximately equivalent to that of the best performing algorithm Phenograph (KMD: 0.926 accuracy, 0.885 NMI, 0.894 ARI; Phenograph: 0.924 accuracy, $0.899 \mathrm{NMI}, 0.925 \mathrm{ARI}$ ). Overall, we find that in contrast to the competing algorithms, whose performance varies between datasets, KMD clustering consistently performs better than or equivalent to the best performing algorithm on each of the datasets. 

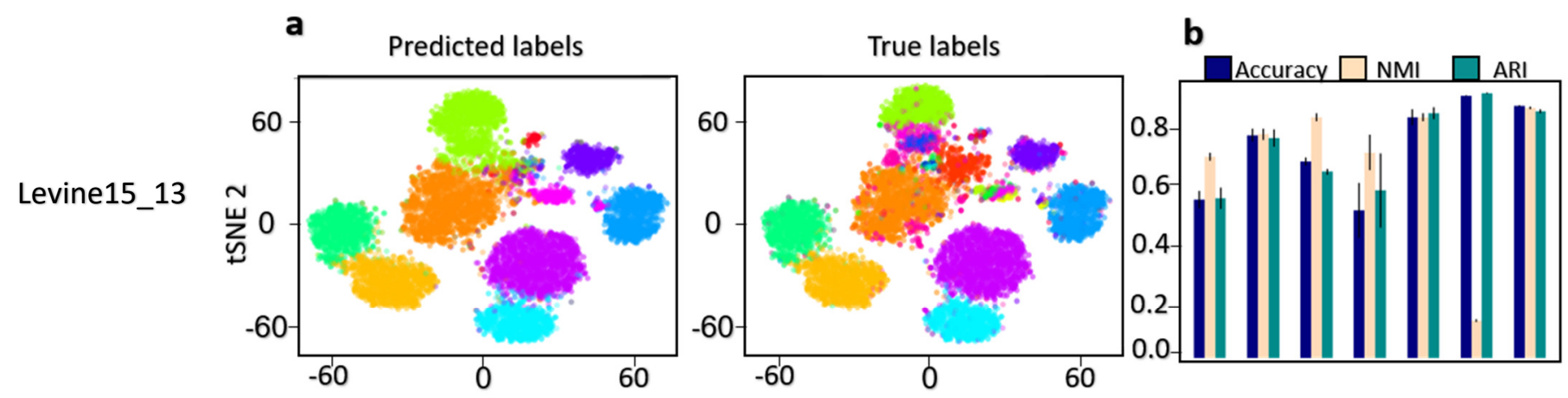

Levine15_32
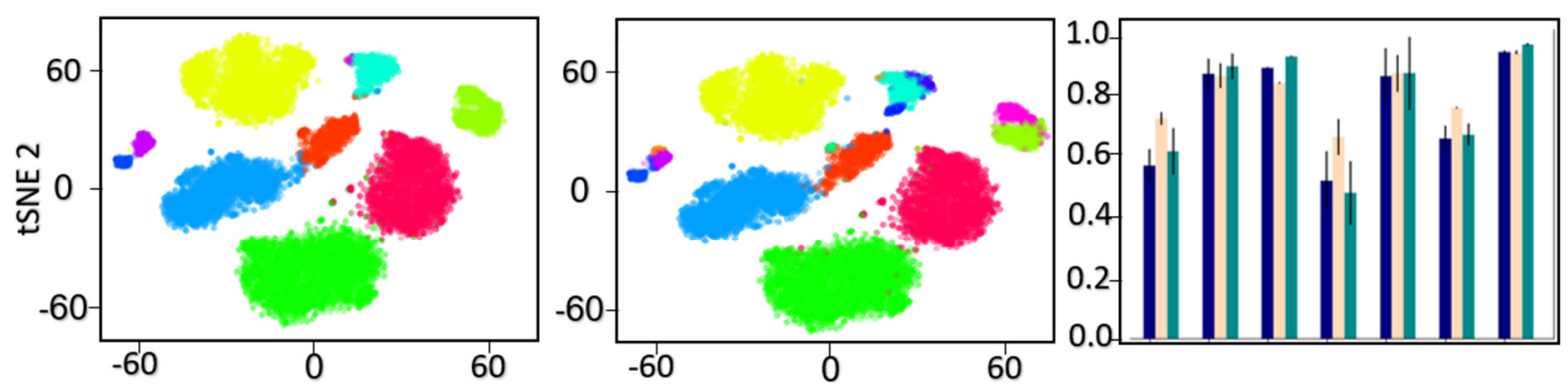

Samusik16

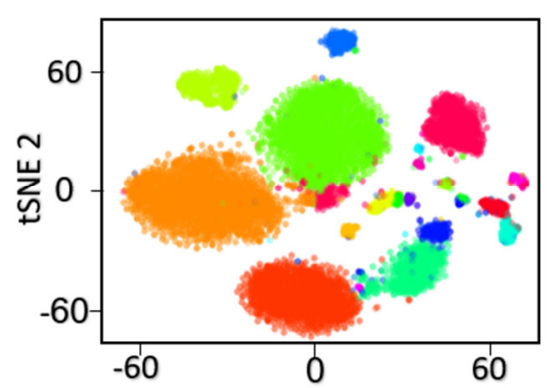

tSNE 1

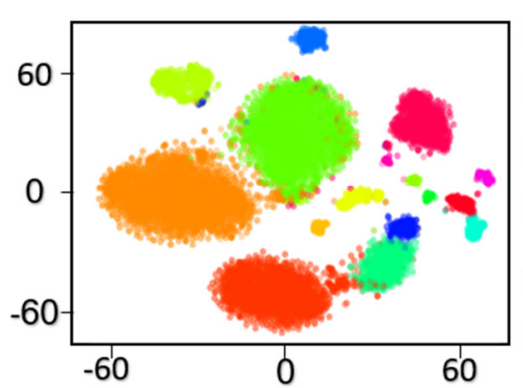

tSNE 1

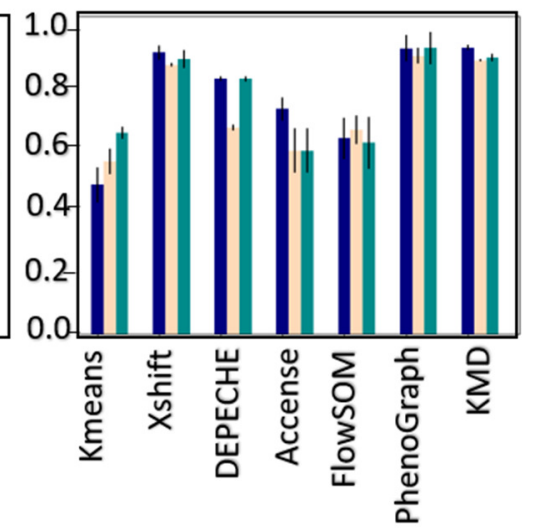

Figure 5. Evaluation on mass cytometry data. (a) tSNE representation of mass cytometry data with clusters colored according to cell type predicted labels (left) and true labels (right) of three datasets: Levine15_13 (20,000 out of 167,044 Cells, 13 markers, 14 clusters), Levine15_32 (20,000 out of 265,627 Cells, 32 markers, 24 clusters), Samusik16 (20,000 out of 86,864 Cells, 44 markers,24 clusters). (b) Average performance of six clustering algorithms: kmeans, Xshift, DEPECHE, Accense, FlowSOM, Phenograph and KMD clustering by accuracy (blue), Normalized Mutual Information (light pink), Adjusted Rand Index (green). Error bars represent standard deviation.

\section{Evaluation on single cell RNAseq data}

Finally, we asked how KMD clustering performs on extremely high-dimensional biological data, in which the number of clustered objects is much smaller than the dimensionality. Unsupervised 
clustering is often used on single cell RNA sequencing data in order to classify cell types based on transcriptional similarity, but these datasets can be challenging to cluster due to the sparse and noisy nature of the data 7 . To assess the performance of KMD clustering, we used three datasets containing gold standard labels: Lawlor17 ${ }^{22}$ (638 cells, 19927 genes, 9 clusters) containing pancreas cells, Zeisel15 23 (3005 cells, 2000 genes, 8 clusters) containing cortex and hippocampus cells and Li17 ${ }^{24}(561$ cells, 57241 genes, 7 clusters) containing human colorectal tumor cells. We note these datasets are relatively small, and are often considered difficult to classify ${ }^{25}$. We then compared the performance of KMD clustering to three methods that were designed for clustering scRNA-seq data: SCAAF, Louvain and Leiden (Scanpy implementation) (Figure 6, Table S4). In all single cell datasets, we used correlation rather than Euclidean distance as recommended in previous studies for this type of data ${ }^{26}$. On the Lawlor17 dataset, KMD outperformed all clustering algorithms (KMD: 0.895 accuracy, $0.796 \mathrm{NMI}, 0.841$ ARI; SCAAF (second best): 0.808 accuracy, 0.760 NMI, 0.0.769 ARI). On the Zeisel15 dataset, KMD outperformed all clustering algorithms (KMD: 0.835 accuracy, $0.780 \mathrm{NMI}, 0.807 \mathrm{ARI}$; SCAAF (second best): 0.711 accuracy, $0.731 \mathrm{NMI}, 0.586 \mathrm{ARI}$ ). On the Li17 dataset, KMD clustering outperformed all clustering algorithms (KMD: 0.938 accuracy, $0.901 \mathrm{NMI} 0.901$ ARI; Louvain(second best): 0.729 accuracy, $0.764 \mathrm{NMI}, 0.592 \mathrm{ARI}$ ). We conclude that KMD outperforms all other tested clustering methods on these scRNA-seq datasets. 
Lawlor17
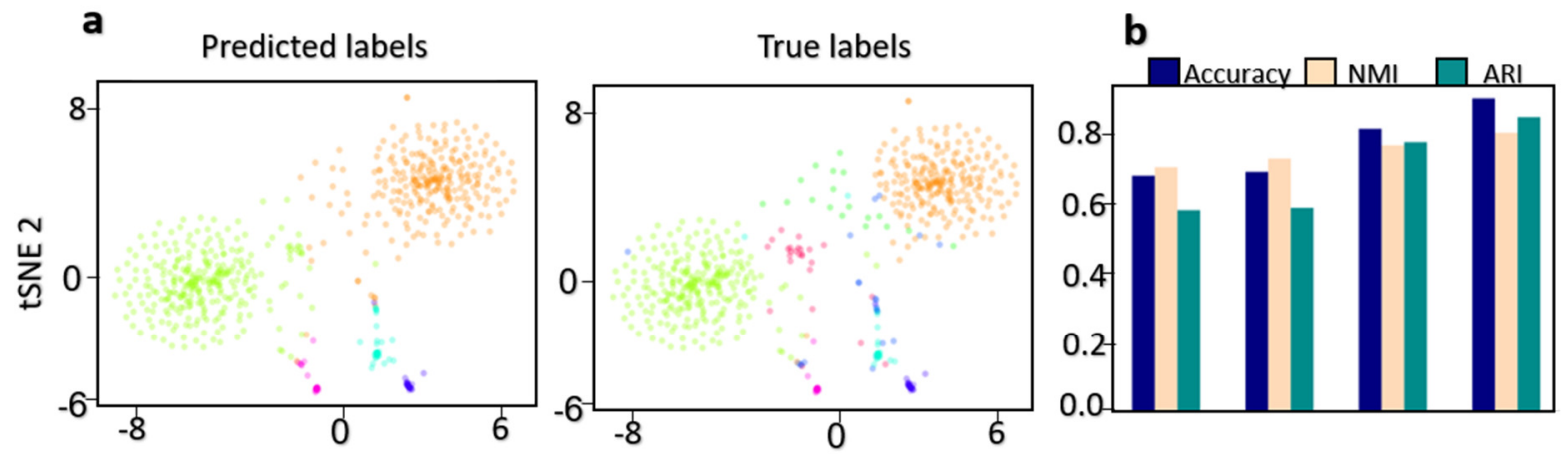

Zeisel15
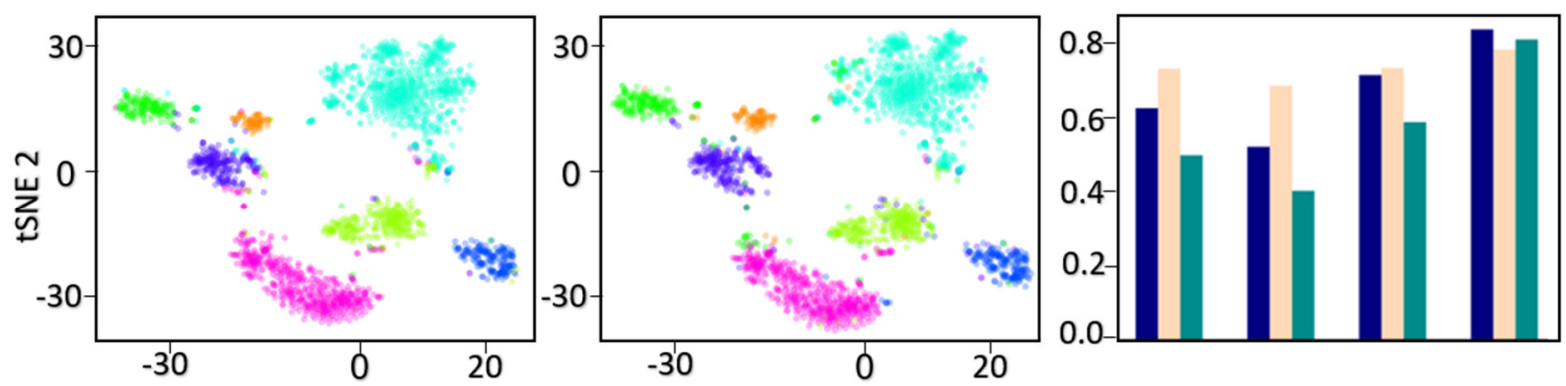

Li17

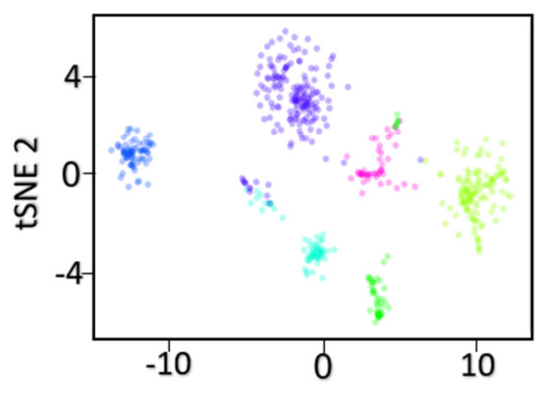

tSNE 1

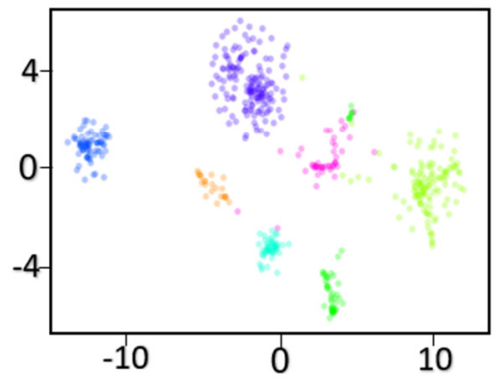

tSNE 1

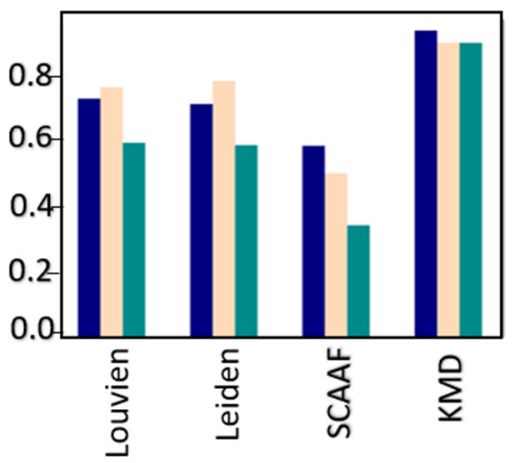

Figure 6. Evaluation on scRNA-seq datasets. (a) tSNE representation of single cell transcriptomics data with clusters colored according to cell type predicted labels (left) and true labels (right) of three datasets: Lawlor17 (638 cells, 19927 genes,9 clusters), Zeisel15 (3005 cells, 2000 genes, 8 clusters) and Li17 (630 cells, 57241 genes, 7 clusters). (b) Performance of four clustering algorithms: Louvien, Leiden, SCAAF and KMD clustering accuracy (blue), Normalized Mutual Information (light pink), Adjusted Rand Index (green).

\section{Discussion and summary}

Average linkage and single linkage hierarchical clustering are well-established generic clustering algorithms. Although they share the same framework, the difference in the definition of linkage 
often leads to dramatically different results from these two algorithms ${ }^{17}$. Although it is known that in machine learning there is no free lunch, we attempted to capture the best properties of both linkages by proposing a novel generalized linkage called KMD linkage. The main challenge arising from this strategy is that it requires to choose the value of the cryptic hyperparameter $k$, the number of minimal intercluster distances to average when calculating pairwise cluster distance (linkage). In unsupervised learning settings, cryptic hyperparameters pose a major hurdle ${ }^{7}$, because the inability to correctly estimate them a priori often leads to post hoc fitting and thus to hidden overfitting. We were thus motivated to find a way of effectively eliminating this hyperparameter by using some kind of intrinsic function which would be predictive of clustering performance without using any external labels. The silhouette score is often used to evaluate and compare clustering solutions, yet it is biased towards globular cluster shapes and is thus not adequate. We speculated that this tendency towards globular clusters may arise from the fact that in the silhouette score, the distance from an object to a cluster is calculated as the average of all pairwise distances ${ }^{20}$, which is effectively average linkage. Thus we considered a modified function, which we call KMD silhouette, where the distance from an object to a cluster is calculated as the $k$ minimal distances, and the value of $\mathrm{k}$ is set to match the value of $k$ used to calculate the KMD linkage. This may seem odd, since it means that the way of scoring the clustering results is different for each value of $k$ and that two $k$ values which yield the same clustering results might be assigned different scores. We also added a term that penalizes large $k$ values, since larger $k$ values artificially inflate the score. Remarkably, we find that our proposed KMD silhouette function predicts clustering performance across $k$ values very well. Given these results, the main hyperparameter $k$ is effectively eliminated by running the clustering across a range of $k$ values (in all cases we limited $k<100$ ) and automatically selecting the solution with the highest KMD silhouette score.

The clustering performance of advanced or specialized clustering algorithms, all of which have cryptic hyperparameters (often multiple ones), can vary greatly even on data of the same type ${ }^{1,7,10 .}$ For example, results from the benchmark study by Liu et al. ${ }^{10}$ on mass cytometry data, which we utilize in the current paper, show that the best competing algorithm in each mass cytometry dataset typically performed poorly on some other mass cytometry dataset. This stands in contrast to KMD clustering which performed well (best or tied for best) across all three datasets, although it was not specifically tailored to mass cytometry data. We speculate that the variation in the performance of other algorithms may be in part due to fitting of cryptic hyperparameters. 
Noise and outliers in data pose a challenge for clustering algorithms, especially in biological data. While single linkage is especially prone to noise due to its calculation being based on a single distance $^{16}$, average linkage is also relatively sensitive to noise as a single incorrect merge of an outlier can have fatal consequences in later clustering stages ${ }^{16}$. Indeed, some clustering algorithms such as DBSCAN have been specifically designed to deal with the challenge of noisy data $^{27}$. In addition to our new linkage strategy in automatic parameter selection, we introduce a simple scheme for dealing with outliers, based on the assumption that tiny clusters which are smaller than a size threshold can be considered to be outliers. Remarkably, we find that KMD clustering performs extremely well with noisy data, outperforming algorithms such as DBSCAN in scenarios where noise was added to such a level that it is even difficult to discern the clusters visually in two dimensions.

Similarly to many other clustering algorithms, KMD clustering requires that the user specify the number of clusters in the data. One could argue that in some cases the user may not have sufficient prior knowledge to estimate this number. Indeed, some clustering algorithms which we compared to do not take the number of clusters as input and instead estimate the number of clusters de novo. However, in many cases it is not clear that there is a single number that is correct, given that clusters may have several levels of granularity ${ }^{17}$. For example, cell types may often belong to larger type groups, or alternatively have subtypes ${ }^{7}$. We thus chose to leave the number of clusters to be determined by the user. However, KMD clustering could potentially estimate the number of clusters, and/or the outlier cluster size threshold by building on the KMD silhouette that we proposed.

While KMD clustering performed remarkably on the tested datasets, we note a number of speed limitations of our approach. First, we note that automatic selection of the hyperparameter $\mathrm{k}$ requires running the clustering with a large range of $k$ values and selecting the solution with the best KMD silhouette score. While these can be run in parallel, it does require more computational resources than a typical simple clustering approach such as standard average linkage clustering. Second, our current implementation is in python/scipy. This makes the code more accessible to users, but results in longer running times then widely used clustering algorithms that are implemented with non-interpreted languages and carefully optimized. Finally, even when disregarding language of implementation, a single run of KMD clustering may be inherently slower than some other approaches (such as single and average linkage clustering) as it requires maintaining for each pairs of clusters a list of the $k$ minimal distances between them. Taken 
together, these factors may prohibit the application of this approach, at least in its current implementation, on very large datasets (e.g. over 100,000 objects).

In conclusion, KMD clustering is a generic clustering algorithm combining novel approaches for generalized linkage approach, automatic hyperparameter selection using an intrinsic function, and outlier-aware partitioning. Compared to a set of tested generic and specialized algorithms, KMD clustering exhibits top performance across a variety of datasets including extreme noise and high dimension, without dataset-specific tuning of hyperparameters.

\section{Methods}

\section{$\underline{\text { KMD clustering algorithm }}$}

The input to the KMD clustering algorithm is a $n \times m$ matrix ( $2 \mathrm{~d}$ ndarray) where $n$ is the number of objects and $m$ is the object dimensionality. Based on the input matrix, a $n \times n$ symmetric distance matrix ( $2 \mathrm{~d}$ ndarray) $D$ between all pairs of objects is computed. The default distance metric is the Euclidean norm. Next, the pairwise distances are used to populate a heap that can efficiently pop the two nearest clusters. Finally, a symmetric matrix $A$ containing the $\mathrm{k}$ minimal distances between every pair of clusters is initialized. $A$ is a $n \times n$ matrix ( $2 \mathrm{~d}$ ndarray) of pointers to lists and is initialized such that $A_{i, j}$ is a list containing the distance between objects $i$ and $j$. Output is stored in array $Z$.

Every iteration of the algorithm includes the following:

1. $x, y=$ get_minimum(heap, $D)$ \# Return indices of two nearest clusters

2. $A_{x,{ }^{*}}=A_{*, x}=\operatorname{merge}\left(A_{x,{ }^{*},} A_{y,{ }^{*}}\right) \quad$ \# Construct the $\mathrm{k}$ minimal distances of the new cluster to every other cluster by merging the respective $k$ minimal distances of $x$ and $y$ to every other cluster in order. Replace cluster $x$ with the new cluster.

3. $D_{x^{*}}=D^{*}, x=\operatorname{mean}\left(A_{x,{ }^{*}}\right) \quad$ \# Update distance matrix

4. $D_{y,{ }^{*}}=D_{*, y}=A_{y,{ }^{*}}=A_{*, y}=$ null \# Eliminate cluster $\mathrm{y}$

5. Z.append $([x, y, \operatorname{dist}(x, y)])$

6. heap.update $\left(A_{x,{ }^{*}}\right) \quad$ \# Update the heap with the new distances

The heap implementation is a modification of the Generic_Linkage algorithm described $\mathrm{in}^{28}$. in which candidates for nearest neighbors of clusters are maintained in a priority queue to speed up the search for the two nearest clusters.

Step 2 is a critical step since in general, updating the distances of a new cluster with an arbitrary linkage can be very inefficient. However, with KMD linkage an efficient update can be performed 
in $\mathrm{O}(n k)$. To see this, consider finding $\operatorname{KMD}(z, i)$ - the $\mathrm{KMD}$ linkage between a new cluster $z$, which was created by merging $x$ and $y$, and an existing cluster $i$. Since $\operatorname{KMD}(z, i)$ must be a subset of $\operatorname{KMD}(x, i) \cup \operatorname{KMD}(y, i)$, we only need to search $2 k$ distances. Furthermore, if we start with lists of length 1 and update by merge-sorting the lists and taking the minimal k elements, the update will only take $\mathrm{O}(k)$ for a pair of clusters, and therefore $\mathrm{O}(n k)$ for all clusters.

In addition, naively one might expect matrix $A$ to require $\mathrm{O}\left(n^{2} k\right)$ space, since we must maintain the $\mathrm{k}$ minimal distances for all pairs of clusters. However, we are able to avoid this with the aforementioned implementation of $A$ as an array of list pointers. To see this, consider that initially $A$ holds only lists of length 1 , and thus requires $\mathrm{O}\left(n^{2}\right)$ space. At each iteration, rows $A_{x,{ }^{*}}$ and $A_{y,{ }^{*}}$ are merged as explained above to create a new row, and then rows $A_{x,{ }^{*}}$ and $A_{y,{ }^{*}}$ are effectively

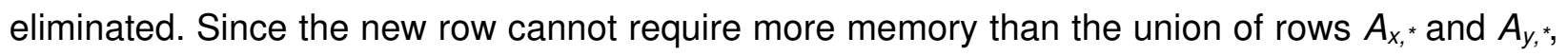
the required space cannot surpass $\mathrm{O}\left(n^{2}\right)$, and actually decreases during the process. This type of implementation is critical, as it allows running the algorithm with large $k$ values, which would be impractical if one was to maintain an array of size $O\left(n^{2} k\right)$.

\section{Outlier-aware partitioning}

Once all clusters are merged, the resulting binary tree is used to apply outlier-aware partitioning. We start with parameters $c$ (the number of clusters) and $m$ (the minimal cluster size / outlier size threshold). Starting from the root of the tree, we first find the first $c-1$ merges where both clusters have a size of at least $m$. The $c$ clusters included at these merges are defined as core clusters. Any objects which are not present in the core clusters are defined as outliers. Note that in general this partitioning is not a fixed-height tree cut as the selected merges can occur at different heights. As shown in Figure S1, clustering results are typically robust to the choice of this parameter. In general, $m$ can be chosen to be slightly smaller than the size of the smallest expected cluster. If no a priori information is available, we suggest setting $m=\max \left(2, n /\left(10^{\star} c\right)\right)$.

In order to assign each outlier to one of the core clusters, we calculate the KMD linkage of the outlier to each of the core clusters and assign it to the core cluster with the minimal distance. In order to provide a confidence level to this assignment, we define a confidence score that compares the KMD distance between the outlier $v$ and its two nearest core clusters $C_{1}$ (nearest cluster) and $C_{2}$ (second nearest cluster):

$$
\operatorname{confidence}(v)=1-\frac{\operatorname{dist}\left(v, C_{1}\right)}{\operatorname{dist}\left(v, C_{1}\right)+\operatorname{dist}\left(v, C_{2}\right)}
$$


The confidence score ranges between 0.5 (lowest confidence) to 1 (highest confidence). all comparisons of clustering performance in the paper were done using these outlier assignments, such that no objects were left out unless stated otherwise.

$\underline{\text { KMD silhouette }}$

The KMD silhouette is an intrinsic function, inspired by the silhouette function, that is used to assess the quality of a clustering solution. The core component of the silhouette is the difference between $a_{i}$, the average distance of object $i$ to the other objects of the assigned (nearest) cluster, and $b_{i}$, the average distance of object $i$ to the objects of the second nearest cluster. Let us denote this difference $d_{i}=b_{i}-a_{i}$. In KMD silhouette we use the same quantity $d_{i}$, except that we define $a_{i}$ and $b_{i}$ slightly differently by using the average of the $k$ minimal distances rather than all distances. For every run $t$ of the clustering algorithm with a $k$ value of $k_{t}$, we calculate the average of these differences and denote this as $s_{t}$, i.e. $s_{t}=\frac{1}{n} \sum_{i} d_{i}^{t}$. Finally, the KMD silhouette of run $t$ is defined as:

$$
\operatorname{KMDsilhouette}(t)=\sqrt{\frac{s_{t}-\min _{i}\left(s_{i}\right)}{\max _{i}\left(s_{i}\right)-\min _{i}\left(s_{i}\right)}}-\frac{k_{t}}{n}
$$

\section{Clustering evaluation}

In order to perform clustering evaluation in an unbiased manner, we chose to work only with datasets where true labels are known. Following Liu et al., we used three different performance metrics: Accuracy, Normalized Mutual Information (NMI) and Adjusted Rand Index (ARI). We denote the true and predicted labels as vectors of integers $t$ and $p$ in which the $i$-th element represents the true and predicted cluster, respectively.

Accuracy: Given a one-to-one matching between predicted and assigned clusters, the accuracy is defined as

$$
\operatorname{Accuracy}(p, t)=\frac{1}{n} \sum \mathbf{1}_{p_{i}=t_{i}}
$$

where $1_{p_{i}=t_{i}}$ is an indicator function that counts when $p_{i}=t_{i}$. The optimal one-to-one matching that maximizes accuracy was found by using the Hungarian algorithm ${ }^{29}$.

Normalized Mutual Information (NMI): We defined mutual information of $t$ and $p$ as: 


$$
I(t, p)=\sum_{p, t} P_{t p}(t, p) \log \left(P_{t p}(t, p) / p_{p}(p) p_{t}(t)\right)
$$

Where $p_{t}(t)$ and $p_{p}(p)$ are the probability distributions and $P_{t p}(t, p)$ is the joint distribution

$\mathrm{NMI}$ is the more commonly used normalized form:

$$
N M I=\left(\frac{2 I(p, t)}{H(t)+H(p)}\right)
$$

Where $H(t), H(p)$ are the information entropies

$\mathrm{NMI}$ is large if $\mathrm{p}$ is an optimal clustering result. $\mathrm{t}=\mathrm{p}$ corresponds to $\mathrm{NMI}=1$

Adjusted Rand Index (ARI):

Rand index $(\mathrm{RI})$ can be computed using the following formula:

$$
R I=\frac{T P+T N}{T P+F P+F N+T N}
$$

TP - objects in a pair are placed in the same group in $t$ and in the same group in $p$

FP - objects in a pair are placed in the same group in $t$ and in different groups in $p$

$\mathrm{FN}$ - objects in a pair are placed in the same group in $\mathrm{p}$ and in different groups in $\mathrm{t}$

TN - objects in a pair are placed in different groups in $t$ and in different groups in $p$

$\mathrm{ARI}$ is calculated by adjusting $\mathrm{RI}$ using the following scheme:

$$
A R I=\frac{R I-\text { Expected_RI }}{1-\text { Expected_RI }}
$$

Where the Expected_RI is defined as:

$$
\text { Expected_RI }=\frac{(T P+F P)(T P+F N)+(T N+F P)(T N+F N)}{(T P+F P+F N+T N)^{2}}
$$

\section{Evaluation on simulated datasets}

All simulated datasets were generated using scikit-learn random sample generators with 1000 objects and seed $=1$. The nested circles dataset was generated using the make_circles generator with the parameters factor $=0.3$ and noise $=0.05$ (noise $=0.14$ for high noise version). The half moons dataset was generated using the make_moons generator with noise $=0.05$ (noise $=0.24$ for 
high noise version). The anisotropic clusters dataset was generated using a transformed dataset generated by the make_blobs generator. The transformation was the dot product of the dataset with the array [[0.6, -0.6], [-0.4, 0.8]]. The random state was set to 170 (185 for high noise version). The globular clusters dataset was generated using the make_blobs generator. The random state was set to 170 (185 for high noise version) and the standard deviation was set to [1.0,2.5,0.5] ([2.0,2.0,2.0] for high noise version). All datasets objects were standardized.

The noisy half moons dataset used in the analyses shown in Figure1, Figure 2 and Supplementary Figure 2 was generated with parameters noise $=0.24$ and seed=5 (Figure 1) or seed=3 (Figure 2 and Supplementary Figure 2).

In all cases where we did not use default running parameters for competing algorithms, we selected new parameter settings that improved the performance of those algorithms relative to the performance under default settings.

Single linkage clustering was run with the AgglomerativeClustering scikit-learn algorithm, using the non-default parameter connectivity to specify the number of neighbors to use for each dataset: 2 neighbors for nested circles and half moons, 10 neighbors for anisotropic clusters and globular clusters.

Average linkage was run with the AgglomerativeClustering scikit-learn algorithm, using default parameters.

Spectral clustering was run with the SpectralClustering scikit-learn algorithm, using non-default parameters eigen_solver='arpack' and affinity='nearest_neighbors'.

DBSCAN was run with the DBSCAN scikit learn algorithm, using the non-default parameter setting: eps $=0.15$ for nested circles and half moons, eps $=0.18$ for globular clusters, eps $=0.15$ for anisotropic clusters.

Gaussian mixture was run with GaussianMixture scikit learn algorithm, using default parameters. KMD clustering was performed as described (minimal cluster size $=50$ ).

\section{Evaluation on mass cytometry datasets}

The datasets Levine15_13 (167,044 Cells), Levine15_23 (265,627 cells) and Samusik16 (86,864 Cells) are known benchmarking mass cytometry datasets and have been used in previous comparisons of algorithms ${ }^{810}$. The true label annotations are known cell types that were manually gated. The transformed and filtered datasets were downloaded from the "flowrepository" 
repository (http://flowrepository.org/id/FR-FCM-ZZPH) ${ }^{8}$. Cells that were unassigned or had ambiguous annotations were discarded. In the interest of consistency and fairness, clustering results for kmeans, Xshift, DEPECHE, Accense, FlowSOM and Phenograph were taken from ${ }^{8}$. Following Liu et al., we randomly sampled 20,000 cells five times from each dataset, ran KMD clustering (minimal cluster size $=50$ ) and calculated the mean and standard deviation for each performance metric.

\section{Evaluation on single cell RNA-seg datasets}

The Li17 dataset consists of 630 single cells sampled from 7 cell lines, with the expression profiling differing between patients due to intratumoral heterogeneity. The dataset was preprocessed by filtering out the $5 \%$ of highest and lowest expressed genes.

The Lawlor17 dataset consists of single cell transcriptomes of 638 human islet cells obtained from five non-diabetic and three type 2 diabetic cadaveric organ donors. The dataset was preprocessed as suggested in ${ }^{11}$ : Normalization by the natural logarithm of one plus the input array; cells with under 200 expressed genes were filtered out; genes that were expressed in less than 3 cells were filtered out; cells with ambiguous annotations were filtered; each cell was normalized by total counts by all genes.

The Zeisel15 dataset consists single cell transcriptome of 3005 mice cerebral cortex cells. The dataset was preprocessed as suggested in ${ }^{11}$ : Normalization by the natural logarithm of one plus the input array; genes that were expressed in less than 3 cells were filtered out; the 2000 genes with the highest variance were selected for the analysis.

The Louvain and Leiden algorithms were executed with default parameters as follows: First, PCA components were calculated with number of components $=50$; next, neighborhood graph was calculated with number of neighbors=15; finally, Louvain and Leiden clusterings were computed with resolution $=1$.

SCAAF was executed using the SCCAF_optimize_all function with the following parameters: The start optimization point was precomputed Louvain annotation; optimization name (prefix) was set to 'L1' for Lowler17 and Li17, and 'L2' for Zeisel15; the algorithm ran on PCA data calculated as explained above, and the minimum self-projection accuracy was 0.93 . The analysis was run 10 times and scores were averaged in order to eliminate the heterogeneity of the dataset random split to train/test datasets. 
KMD clustering was run with correlation distance as recommended $i^{26}$ and with minimal cluster size of 10 due to the small sizes of the clusters in these datasets.

Availability

KMD clustering is implemented in python using the numpy/scipy and scikit-learn libraries, and is available with documentation at https://github.com/KaplanLab/KMDHierarchicalClustering. The repository includes python code and Jupyter notebooks for reproducing shown results.

\section{Acknowledgements}

We thank Nir Ailon, Shai Shen Orr, Amit Zeisel and members of the Kaplan Lab for helpful discussions and comments. This research was funded by Israel Science Foundation Individual Research Grant 1479/18.

\section{References}

1. Ronan, T., Qi, Z. \& Naegle, K. M. Avoiding common pitfalls when clustering biological data. Sci. Signal. 9, 1-13 (2016).

2. Aouf, M., Lyanage, L. \& Hansen, S. Review of data mining clustering techniques to analyze data with high dimensionality as applied in gene expression data (June 2008). in 5th International Conference Service Systems and Service Management - Exploring Service Dynamics with Science and Innovative Technology, ICSSSM'08 (2008). doi:10.1109/ICSSSM.2008.4598505

3. Kaplan, N. \& Linial, M. ProtoBee: Hierarchical classification and annotation of the honey bee proteome. Genome Res. 16, 1431-1438 (2006).

4. Kaplan, N. \& Linial, M. Automatic detection of false annotations via binary property clustering. BMC Bioinformatics 6, (2005).

5. Kaplan, N. \& Dekker, J. High-throughput genome scaffolding from in vivo DNA interaction frequency. Nat. Biotechnol. 31, 1143-1147 (2013).

6. Kaplan, N., Friedlich, M., Fromer, M. \& Linial, M. A functional hierarchical organization of the protein sequence space. BMC Bioinformatics 5, (2004). 
7. Kiselev, V. Y., Andrews, T. S. \& Hemberg, M. Challenges in unsupervised clustering of single-cell RNA-seq data. Nat. Rev. Genet. 20, 273-282 (2019).

8. Weber, L. M. \& Robinson, M. D. Comparison of clustering methods for high-dimensional single-cell flow and mass cytometry data. Cytom. Part A 89, 1084-1096 (2016).

9. Duò, A., Robinson, M. D. \& Soneson, C. A systematic performance evaluation of clustering methods for single-cell RNA-seq data. F1000Research 7, 1141 (2018).

10. Liu, X. et al. A comparison framework and guideline of clustering methods for mass cytometry data. Genome Biol. 20, 1-18 (2019).

11. Miao, Z. et al. Putative cell type discovery from single-cell gene expression data. Nat. Methods 17, 621-628 (2020).

12. Van Gassen, S. et al. FlowSOM: Using self-organizing maps for visualization and interpretation of cytometry data. Cytom. Part A 87, 636-645 (2015).

13. Levine, J. H. et al. Data-Driven Phenotypic Dissection of AML Reveals Progenitor-like Cells that Correlate with Prognosis. Cell 162, 184-197 (2015).

14. Wolf, F. A., Angerer, P. \& Theis, F. J. SCANPY: Large-scale single-cell gene expression data analysis. Genome Biol. 19, 15 (2018).

15. Satija, R. SEURAT - R toolkit for single cell genomics: single cell integration in Seurat v3.0. satijalab.org. (2015). Available at: https://satijalab.org/seurat/. (Accessed: 7th September 2020)

16. Nielsen, F. Introduction to HPC with MPI for Data Science. in Springer 304 (2016). doi:10.1007/978-3-319-21903-5

17. Dasgupta, S. \& Long, P. M. Performance guarantees for hierarchical clustering. J. Comput. Syst. Sci. 70, 555-569 (2005).

18. Barbakh, W. A., Wu, Y. \& Fyfe, C. Review of Clustering Algorithms. in 7-28 (Springer, Berlin, Heidelberg, 2009). doi:10.1007/978-3-642-04005-4_2

19. Sander, J., Qin, X., Lu, Z., Niu, N. \& Kovarsky, A. Automatic extraction of clusters from hierarchical clustering representations. Lect. Notes Artif. Intell. (Subseries Lect. Notes Comput. Sci. 2637, 75-87 (2003).

20. Rousseeuw, P. J. Silhouettes: A graphical aid to the interpretation and validation of 
cluster analysis. J. Comput. Appl. Math. 20, 53-65 (1987).

21. Pedregosa et al. Scikit-learn: Machine Learning in Python. (2011). Available at: https://scikit-learn.org. (Accessed: 7th September 2020)

22. Lawlor, N. et al. Single-cell transcriptomes identify human islet cell signatures and reveal cell-type-specific expression changes in type 2 diabetes. Genome Res. 27, 208-222 (2017).

23. Zeisel, A. et al. Cell types in the mouse cortex and hippocampus revealed by single-cell RNA-seq. Science (80-. ). 347, 1138-1142 (2015).

24. Li, H. et al. Reference component analysis of single-cell transcriptomes elucidates cellular heterogeneity in human colorectal tumors. Nat. Genet. 49, 708-718 (2017).

25. Fortunato, S. \& Barthélemy, M. Resolution limit in community detection. Proceedings of the National Academy of Sciences of the United States of America 104, 36-41 (2007).

26. Skinnider, M. A., Squair, J. W. \& Foster, L. J. Evaluating measures of association for single-cell transcriptomics. Nat. Methods 16, 381-386 (2019).

27. Stuetzle, W. \& Nugent, R. A generalized single linkage method for estimating the cluster tree of a density. J. Comput. Graph. Stat. 19, 397-418 (2010).

28. Müllner, D. Modern hierarchical, agglomerative clustering algorithms. 1-29 (2011).

29. Samusik, N., Good, Z., Spitzer, M. H., Davis, K. L. \& Nolan, G. P. Automated mapping of phenotype space with single-cell data. Nat. Methods 13, 493-496 (2016). 